\title{
The Design of Desktop Two Rounds of Self-balancing Robot
}

\author{
Zhang Yingkun ${ }^{123, a}$, Hao Cunming ${ }^{123, a}$, Zhen Zhuo ${ }^{4, a}$ \\ ${ }^{1}$ Institute of Applied Mathematics, Hebei Academy of Sciences, Hebei Shijiazhuang 050081 , \\ China \\ ${ }^{2}$ SJZ JKSS Technology Co., Ltd,Hebei Shijiazhuang 050081, China \\ ${ }^{3}$ Hebei Authentication Technology Engineering Research Center,Hebei Shijiazhuang 050081, \\ China \\ ${ }^{4}$ Hebei university of science and technology, Hebei Shijiazhuang 050000, China \\ a735245635@qq.com
}

Keywords: Desktop robot; Self-balancing; PID

\begin{abstract}
In view of the disadvantages of these existing two rounds of self-balancing robots such as large volume, complex control, design a kind of desktop two rounds of self-balancing robot. The microcontroller based on the Arduino is used as the main controller, which uses the MPU - 6050 attitude sensor and speed sensor to get robot Angle and speed parameters, and constitutes the Angle and speed's double closed loop PID control to realize the robot's self balancing control, mobile phone with bluetooth software realizes the wireless control of the robot. The robot is small in size, low cost and reliable performance and can be used as a research experiment platform of advanced control algorithm.
\end{abstract}

\section{Introduction}

With the rapid development of science and technology, the application range of the robot is expanding. Wheeled mobile robot ${ }^{[1]}$ is an important branch of the robot family, because it has advantages of simple structure, low cost, has become a hotspot in the research of the robot. At the same time, the work environment and tasks of the mobile robot is becoming more and more complex, two rounds of self-balancing robot ${ }^{[2,3]}$ arises at the historic moment, the robot prominent advantage is the ability to achieve the balance, and flexible, adaptable to the complicated environment, its typical applications include space exploration, dangerous goods transport, security patrol and environmental exploration, etc.

However, the traditional two rounds of self-balancing robot is mostly large, its quality is heavier, and has higher requirements to the control strategy, which limits its application scope. Therefore, based on the analysis and summary of the existing large two-wheeled self-balancing robot such as SegWay, this paper designs a kind of desktop two rounds of self-balancing robot, which has small volume, flexible in movement, low driving power, can run for a long time, and so on.

\section{The design of robot overall structure}

Desktop two-wheeled self-balancing robot is constituted by based on the Arduino microcontroller, posture sensor, speed sensor, bluetooth module and motor drive circuit and so on, the overall structure diagram is shown in figure 1. The structure design of two-wheeled self-balancing robot has a great influence on the control algorithm, its body height and center of gravity location are the important factors that affect the control algorithm complexity, only to ensure the reasonable structure of the robot, in order to realize the robot's self balancing.Therefore, this paper uses hierarchical design ideas to build desktop type two rounds of self-balancing robot, the layer from top to bottom is control layer, the power layer and the executive layer, as shown in figure 2. 


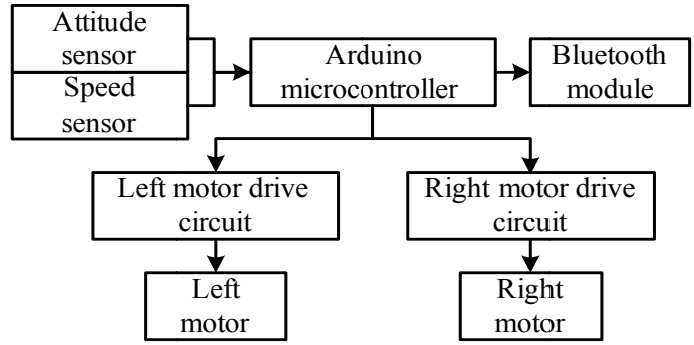

Fig 1 the overall structure diagram of Robot balancing robot layered structure

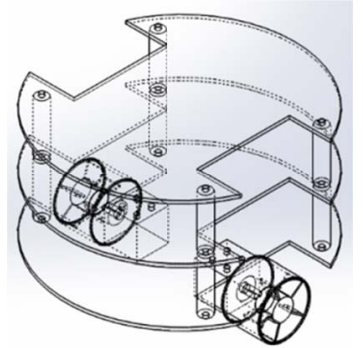

Fig 2 desktop two rounds of selfdesktop two rounds of self-balancing Hardware structure

The main controller. The main controller this article choosed is a microcontroller based on the Arduino, the development board is based on the latest Arduino hardware platform Arduino $\mathrm{UNO}^{[4]}$, the integration of the microprocessor is ATmega328.ATmega328 has $32 \mathrm{~KB}$ FLASH, at the same time, also has $2 \mathrm{~KB}$ SRAM and $1 \mathrm{~KB}$ block of EEPROM, its storage spaces are very rich.The development board has 14 digital input/output IO, six of them can provide 8 PWM output; each of six analog input IO has the resolution of the 10; it has the SPI and I2C communication interface, built-in UART serial communication can be realized through digital IO and the external.

Attitude sensor. Considering the requirement of desktop two rounds of self-balancing robot limits on volume, the attitude sensor this paper choosed is MPU-6050. MPU-6050 is the world's first integrated 6 axis motion processing components, compared to many components, which avoids the combination difference problems between axis of gyroscope and accelerator, and reduces a lot of space ${ }^{[5]}$.MPU-6050 integrates the three axis gyroscope and three axis accelerator, and can be connected to the third party such as accelerator, magnetic sensor, and other sensors through the second I2C port.Digital Motion processing (DMP: Digital Motion Processor) hardware acceleration engine output the complete fusion calculation results of 9 shaft by the $\mathrm{I} 2 \mathrm{C}$ port in the form of a single data flow.The processing database of InvenSense can handle complex movement test data, and reduces the movement of dealing with the operation of the operating system loads, and provides a framework for application development API.MPU-6050 internal integrated temperature sensor can realize data correction.

The bluetooth module.On the basis of realization balance of the robot, in order to enhance the maneuverability of robot, the desktop two rounds of self-balancing robot increases the bluetooth operation, the industrial-grade master-slave integrated bluetooth module BC -04 is selected. This module has the advantages of small volume, reliable performance, convenient interface.In control, bluetooth module $\mathrm{BC}-04$ just receives control instruction from the phone client, so $\mathrm{BC}$ - 04 is set to pattern from the machine.

Driver module. In this paper, the drive module adopts dc gear motor, reduction ratio is 1:48, rated voltage is $3 \sim 9 \mathrm{v}$. They not only can well control the speed of dc motor, and the output torque has also improved.This article uses the dc deceleration motor for high anti-interference, compared with the ordinary motor without interference, its internal joined the varistor, can remove the instantaneous interference of power supply when motor starts, and has more stable during working. The typical $\mathrm{H}$ bridge driving circuit is adopted to control the two way dc gear motor by PWM, the input signal using PWM signal to control, so as to realize the motor speed adjustment, as shown in figure 3.

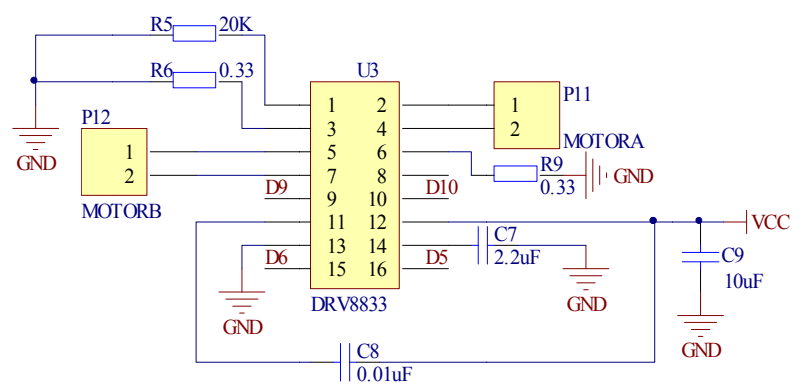

Fig $3 \mathrm{H}$ bridge driving circuit 


\section{Double closed loop PID balance control}

PID control is classical control strategy in the field of motion control, this paper adopts double closed loop PID control to realize the robot self balancing control, algorithm implementation process as shown in figure 4.The outer loop PID for angle ring is to keep robot body angle of 0 degree. The inner loop PID for speed loop is through control dc motor output to make the robot wheel speed and Angle is consistent with the output value.In the diagram,the parameter $U$ expectations for robot body tilt angle, the parameters of the theoretical value is always 0; the parameter $\mathrm{V}$ expectations dc motor speed for robot; the parameter $\mathrm{X}$ expectations robot actual measuring wheel motor speed; parameter $\mathrm{Y}$ is the actual measure tilt angle of robot.

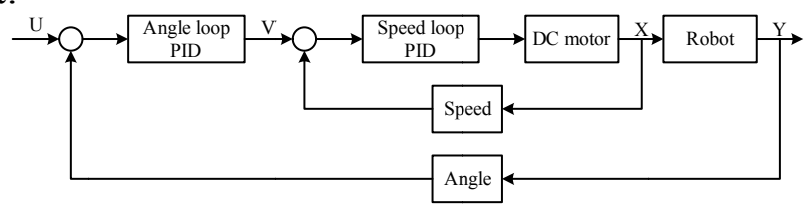

Fig 4 double closed loop PID control

\section{Bluetooth software on mobile phone}

This paper develops a bluetooth software based on Android mobile phone, to realize to control the desktop two rounds of self-balancing robot remote by wireless control, development tools used is the Eclipse integrated development environment.The working process of bluetooth software is shown in figure 5 and figure 6 is the bluetooth software interface.

The operation steps of bluetooth software on mobile phone are as follows:

Open the bluetooth software on mobile phone, click "connect", it needs to scan for devices before using at the first time, then chooses the equipment named "Robot" to connect. Paired with mobile phone connection for the first time need to enter a pairing code, the default value is "1234", and after click the button "ok", the bluetooth software on mobile phone will pair with and connect to $\mathrm{BC}-04$; You can through the arrow keys to control the robot's driving condition. It is important to note that the motion of the robot by pressing the length of the distance and buttons, it means that the robot can be keep working accordance with the instructions only when the button is pressed, if the button is loosen the robot will stop the movement.

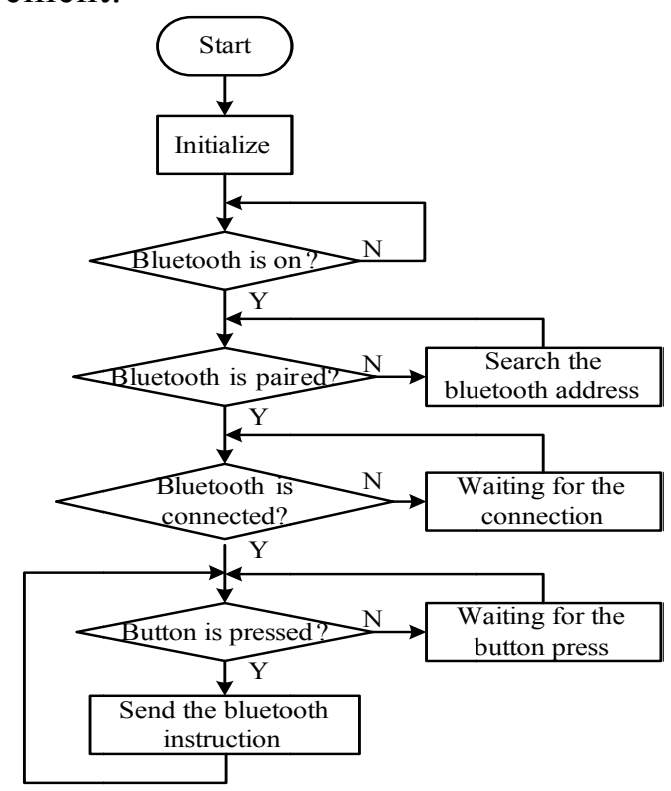

Fig 5 working process of bluetooth software

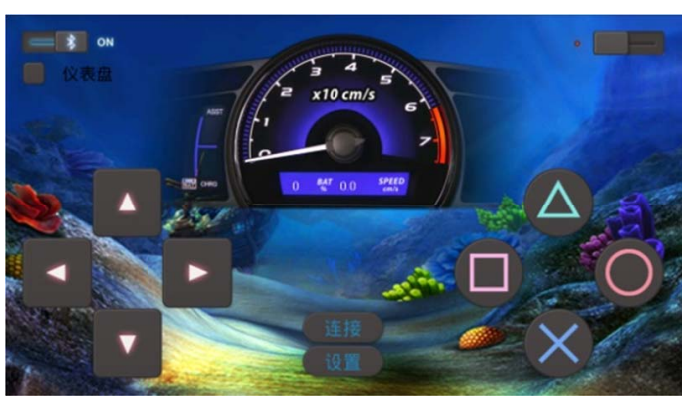

Fig 6 bluetooth software interface 


\section{Conclusion}

On the basis of summarizing the advantages of existing two rounds of self-balancing robots and meet the desktop features, the physical system of desktop two rounds self-balancing robot is designed in this paper. Using MPU-6050 to implementation of the balance of the robot posture detection, which not only saves development cost, but also avoids the complex calculation data fusion. The double closed loop PID control can improve the control precision, and make robot keep a steady equilibrium profile. The bluetooth software on Mobile phone is developed to implement the wireless control of the robot.The robot can keep stable performance in practical applications, and be applied in more places with machine vision system in the future.

\section{Reference}

[1] Li Renhou. An introduction of autonomous mobile robot[M]. Xi 'an: Xi 'an jiaotong university press, 2006.

[2] Gan Jiafei. The two-wheeled self-balancing robot system design and motion control research[D].Bei Jing: Beijing university of technology,2010.

[3] Li Shizhen. The motion control research of flexible two-wheeled self-balancing robot[D].Bei Jing: Beijing university of technology, 2011.

[4] Shen Jinxin. The development combat of Arduino and Labview [M]. Bei Jing: Mechanical industry press, 2014.

[5] Li Fei. The research of four rotor aircraft attitude self balancing control system[D].Hei Longjiang: Harbin institute of technology, 2013.

Author introduction:

Zhang Yingkun (1988 -), male (han), hebei shijiazhuang, intern researcher, master degree, main research direction of intelligent control algorithm and parallel manipulator motion control. 